\title{
La Satisfaction Dans Les Études : Validation De L'échelle De Vallerand Et Bissonnette (1990) Et Son Impact Sur Le Travail Scolaire Perçu Des Lycéens
}

\author{
Bawa Ibn Habib, PhD
}

Université de Lomé, Département de Psychologie Appliquée, Togo

Moumoula Issa Abdou, PhD

Université de Norbert Zongo, Département de Psychologie, Burkina Faso

Doi:10.19044/esj.2018.v14n31p265 URL:http://dx.doi.org/10.19044/esj.2018.v14n31p265

\begin{abstract}
This study not only attempts to validate a cross-cultural measure of satisfaction in Canadian-inspired high school studies among Togolese students, but also to ascertain the effects of academic satisfaction on their perception of their school work. To achieve this double objective, 644 high school students have agreed to participate in the survey and to appreciate their school work. The results relating to the psychometric properties of the scale support those obtained by the authors during its construction. In addition, school satisfaction has been foundational in the psychogenetic understanding of school work.
\end{abstract}

Keywords: school satisfaction, psychometric properties, school work

Résumé

Cette étude non seulement tente de valider un instrument transculturel de mesure de la satisfaction dans les études au secondaire d'inspiration canadienne auprès des élèves togolais mais aussi de s'assurer des effets de la satisfaction scolaire sur la perception qu'ils ont de leur travail scolaire. Pour atteindre ce double objectif, 644 lycéens ont bien accepté de participer à l'enquête et d'apprécier leur travail scolaire. Les résultats relatifs aux propriétés psychométriques de l'échelle soutiennent ceux obtenus par les auteurs au cours de sa construction. En plus, la satisfaction scolaire s'est avérée fondamentale dans la compréhension psychogénétique du travail scolaire.

Mots-clés : satisfaction scolaire, propriétés psychométriques, travail scolaire 


\section{Introduction}

Dans le monde entier, les systèmes éducatifs se proposent d'offrir aux apprenants une formation de qualité. Au Togo, toutes les bonnes intentions du gouvernement en faveur du secteur de l'éducation sont contenues dans la Politique Sectorielle de l'Éducation (PSE) qui est axée sur trois points : accroissement de l'accès, amélioration de la qualité et renforcement des capacités institutionnelles. C'est justement sur la base de cette politique que les actions concrètes suivantes sont posées : la gratuité de l'école primaire, la discrimination positive des frais de scolarité au secondaire en faveur des filles, la formation des enseignants presque chaque année dans les écoles normales, la construction des infrastructures scolaires... Il est indéniable que toutes ces bonnes actions devraient rejaillir sur la qualité de la formation et produire chez les apprenants une satisfaction certaine qui se traduirait par l'amour pour leur école et la réussite ou le bon travail scolaire.

Cette étude vise à valider un instrument transculturel de mesure de la satisfaction dans les études au secondaire d'inspiration canadienne auprès des élèves togolais. Elle a aussi l'objectif des'assurer des effets de la satisfaction scolaire sur la perception qu'ils ont de leur travail scolaire.

Pour atteindre ce double objectif, le cadre conceptuel permettra de clarifier la notion de satisfaction scolaire et revisiter la littérature à propos du lien entre cette variable et les performances scolaires. À la suite de la description de l'approche méthodologique, les résultats de la validation et de la relation supposée seront présentés, suivis enfin d'une comparaison de ces résultats avec ceux de la littérature et les perspectives futures. Mais avant tout, il est important de comprendre le sens que nous donnons aux notions de satisfaction scolaire et de travail scolaire perçu.

En effet, avant de définir la notion de satisfaction scolaire, une clarification préalable de celle de satisfaction est nécessaire. Elle est "l'attitude d'une personne à l'égard d'un objet. Elle constitue une combinaison complexe de cognitions (croyances ou savoirs), d'émotions (sentiments, dispositions ou jugements) et de tendances comportementales » (Hamner et Organ, 1978, cités par Aldemir et Gülcan, 2004, p. 123). Elle peut concerner n'importe quel objet. C'est pourquoi, «lorsqu'une personne déclare qu'elle est satisfaite de quelque chose, on considère qu'elle a une attitude positive à l'égard de cet objet. Inversement, une personne peut être insatisfaite de quelque chose. Dans ce cas on considère qu'elle a une attitude négative à l'égard de cet objet » (Aldemir \& Gülcan, 2004, idem). Ce qui est sûr, c'est que la satisfaction est une notion subjective et dépend de chaque individu.

En milieu scolaire, la satisfaction est « une représentation cognitive et affective de l'atteinte des objectifs visés » (Vallerand \& Bissonnette, 1990, p. 296) par un élève ou un étudiant. Pour les mêmes auteurs, la satisfaction scolaire fait, par conséquent, partie de la dimension affective de l'engagement 
scolaire. Pour Randolph et al. (2009, cités par Gaudonville, 2017), la satisfaction scolaire est l'un des quatre construits qui sous-tendent la satisfaction de la vie (elle-même facteur de la qualité de vie ou du bien-être subjectif) des enfants et des adolescents dans le domaine scolaire. Il s'agit d'une dimension affective concernant les sentiments de l'élève à l'égard de l'école et la perception qu'il en a : le fait d'aimer l'école, le sentiment d'appartenance, l'intérêt et la motivation pour les apprentissages, l'ambition, le sens donné à l'école, la relation aux enseignants... (Robin \& Roux, 2013).

Une telle définition présage, d'après Vallerand et Bissonnette (1990), « une intime relation [...] entre la motivation et la satisfaction vis-à-vis des études, ainsi qu'entre cette dernière et [...] la performance scolaire, l'apprentissage et la persévérance dans les études » (p. 296). C'est justement la relation entre la satisfaction scolaire et la performance scolaire qui nous intéresse dans cette étude car en réalité, le travail scolaire renvoie à la perception que les élèves eux-mêmes ont de leur performance scolaire.

Il faut reconnaître que peu d'études ont été faites sur le sujet selon Vallerand et Bissonnette (1990). Ces quelques rares travaux qui sont à notre suffrage sont parvenus à des conclusions mitigées.

À l'issue de l'étude de Huebner (1991 rapportée par Gaudonville, 2017), portant sur les performances scolaires (note moyenne en lecture, orthographe, mathématiques et sciences) et le score à la Students'Life Satisfaction Scale (SLSS), les résultats montrent que les performances scolaires ne sont pas corrélées de manière significative avec la satisfaction de vie globale (Huebner \& Alderman, 1993). Dans la même ligne, McCullough et Huebner (2003) aboutissent à la conclusion que la satisfaction de vie globale en contexte scolaire n'est pas liée aux performances scolaires.

Des autres travaux comme ceux de Bean et Bradley (1986) et Pike (1991) convergent vers des résultats contraires. En effet, ces auteurs montrent que la satisfaction/l'insatisfaction manifestée à l'égard de l'université/faculté a un impact considérable sur les résultats de l'étudiant. Jorgensen, Fichten, et Havel (2012) rejoignent les auteurs précédents. À l'aide du Student Satisfaction Inventory de Shreiner et Juillerat (1994), ils montrent également à suffisance que la satisfaction globale était plus étroitement liée aux résultats scolaires. Selon Bacro et al. (2014), le bien-être à l'école vu comme une réalité unidimensionnelle correspondrait à la satisfaction scolaire. Sous cet angle, ils démontrent que, dans l'échantillon de collégiens, la satisfaction scolaire et le bien-être ont un impact sur les performances académiques en français, mais pas en mathématiques.

En somme, si les recherches présentées aboutissent à des résultats non convergents, c'est parce que d'une part, certaines s'appuient sur des mesures directes de performances scolaires comme les notes moyennes ou les notes en lecture ou en mathématiques (Bacro et al., 2014 ; Huebner, 1991) ; et d'autres 
sur des indicateurs de difficultés scolaires ou performances issues de tests standardisés (McCullough \& Huebner, 2003). D’autre part l'appréciation de la satisfaction ne se fait pas de la même manière. Lorsque certains chercheurs utilisent des outils unidimensionnels (Bacro et $a l ., 2014$ ) d'autres utilisent des outils multidimensionnels (McCullough \& Huebner, 2003). Pour la plupart de ces outils, leur validité et leur fidélité demeurent incertaines (Vallerand \& Bissonnette, 1990). Toutes ces raisons nous réconfortent dans notre intention de valider l'échelle de satisfaction dans les études avant son utilisation pour vérifier la relation entre la satisfaction dans les études et les performances ou travail scolaire perçu des lycéens togolais. À cet effet, nous postulons, d'une part, que cette échelle présente des qualités psychométriques satisfaisantes et, d'autre part, que plus les élèves sont satisfaits dans leurs études, plus ils perçoivent leur travail scolaire comme bon. Dans les lignes qui suivent, nous décrivons l'approche méthodologique qui a permis de conduire cette étude.

\section{Méthodes}

\subsection{Participants}

Au total six cent quarante-quatre lycéens fréquentant neuf lycées de la ville de Lomé ont accepté de répondre à nos questionnaires. Ils sont issus à la fois des séries d'études littéraire $\mathrm{A}$, scientifique $\mathrm{C}$ et scientifique $\mathrm{D}$. Ils ont été sélectionnés à l'aide de la technique du «tout-venant». Conformément au tableau ci-dessous, on compte plus d'adultes $(53,4 \%)$ que d'adolescents (46,6\%). L'âge moyen est de 19 ans. En plus, les garçons sont plus nombreux que les filles $(59,3 \%$ contre 40,4\%). Enfin, les élèves de la série scientifique D représentent plus de la moitié de l'effectif total.

Tableau 1. Caractéristiques des participants

\begin{tabular}{|r|c|c|}
\hline $\begin{array}{c}\text { Caractéristiques des } \\
\text { participants }\end{array}$ & Effectifs & Pourcentage \\
\hline \multicolumn{2}{|c|}{ Age $(N=644)$} \\
\hline Adolescents & 300 & 46,6 \\
\hline Adultes & 344 & 53,4 \\
\hline \multicolumn{2}{|c|}{ Sexe $(N=642)$} \\
\hline Masculin & 382 & 59,3 \\
\hline Féminin & 260 & 40,4 \\
\hline Série d'études $(N=644)$ \\
\hline Littéraire A & 269 & 41,8 \\
\hline Scientifique C & 10 & 1,6 \\
\hline Scientifique D & 365 & 56,7 \\
\hline
\end{tabular}

\subsection{Mesure des variables}

\subsubsection{Variable indépendante}

La variable indépendante dans cette étude est « la satisfaction dans les études ». Elle est mesurée à l'aide de l'Echelle de Satisfaction dans les Etudes (ESDE) de Vallerand et Bissonnette (1990). Il s'agit d'une échelle destinée « à 
mesurer une évaluation générale et subjective de la satisfaction scolaire en regard avec les critères personnels de l'étudiant. [...] relativement courte [...] formulée de façon simple [...] de sorte à éviter un effet de plafonnement dans les réponses » (p.298). Elle est unidimensionnelle. L'ESDE est composée de 5 items dont les réponses à entourer s'étalent sur une échelle de Likert en 7 points allant de 1 (fortement en désaccord) à 7 (fortement en accord) tel que le montre le Tableau 1 ci-dessous. À l'issue de la pré-enquête, aucune difficulté n'a été soulevée par les élèves concernant la compréhension des items. Par contre, il se fait qu'ils ne parvenaient pas à nuancer les 7 valeurs de l'échelle de Likert indiquées par les auteurs. Ils ont proposé de les réduire à 5 points allant de 1 (en désaccord) à 5 (en accord). Sur cette enquête, les scores obtenus varient entre le score minimal 5 et le score maximal 25.

Tableau 2. Echelle de Satisfaction Dans les Études (Vallerand \& Bissonnette, 1990)

\begin{tabular}{|c|c|c|c|c|c|c|}
\hline $\begin{array}{c}\text { Fortement } \\
\text { en } \\
\text { désaccord } \\
1\end{array}$ & $\begin{array}{c}\text { En } \\
\text { Désaccord } \\
2\end{array}$ & $\begin{array}{l}\text { Légère- } \\
\text { ment } \\
\text { en } \\
\text { désac- } \\
\text { cord } \\
3\end{array}$ & $\begin{array}{c}\text { Ni en } \\
\text { désaccord } \\
\text { Ni en accord } \\
4\end{array}$ & $\begin{array}{c}\text { Légère- } \\
\text { ment } \\
\text { en accord } \\
5\end{array}$ & $\begin{array}{c}\text { En } \\
\text { accord } \\
6\end{array}$ & $\begin{array}{c}\text { Fortement en } \\
\text { accord } \\
7\end{array}$ \\
\hline \multicolumn{6}{|c|}{ Items } & Entoure \\
\hline \multicolumn{6}{|c|}{$\begin{array}{r}\text { 1. En général, ma vie académique correspond de } \\
\text { près à mes idéaux }\end{array}$} & 1234567 \\
\hline \multicolumn{6}{|c|}{ 2. Mes conditions de vie scolaire sont excellentes. } & 1234567 \\
\hline \multicolumn{6}{|c|}{ 3. Je suis satisfait (e) de ma vie académique. } & 1234567 \\
\hline \multicolumn{6}{|c|}{$\begin{array}{r}\text { 4. Jusqu'à maintenant, j'ai obtenu les choses importantes } \\
\text { que je voulais dans le monde scolaire. }\end{array}$} & 1234567 \\
\hline \multicolumn{6}{|c|}{$\begin{array}{r}\text { 5. Si je pouvais recommencer mes études, je n'y } \\
\text { changerais presque rien. }\end{array}$} & 1234567 \\
\hline
\end{tabular}

Le choix de cette échelle se justifie non seulement par le fait qu'elle est une des rares à exister en français, mais aussi elle présente l'avantage d'être courte ( 5 items au total) et formulée de façon aussi simple qu'elle est utilisable du secondaire à l'université (Vallerand \& Bissonnette, 1990).

\subsubsection{Variable dépendante}

Cette variable concerne le travail scolaire perçu. Il revient à chaque élève de répondre à la question suivante: "Dans l'ensemble, comment trouves-tu ton travail scolaire ?» en opérant un choix entre $1=$ bon travail et $2=$ mauvais travail.

\subsection{Méthodes de traitement des données}

La validité de notre outil de mesure est faite à partir des statistiques suivantes : la courbe de Gauss, l'aplatissement, l'asymétrie et le test $Z$ de Kolmogorov-Smirnov pour la sensibilité, l'alpha de Cronbach pour la fidélité, les analyses factorielles et les matrices de corrélation pour la validité de 
contenu du questionnaire. La validité différentielle et la relation entre la satisfaction dans les études et le travail scolaire perçu sont vérifiées à l'aide des analyses de variances (ANOVA) avec le calcul du t de Student.

\section{Résultats}

\subsection{Validation de l'échelle de Vallerand et Bissonnette (1990)}

\subsection{1. Étude de la sensibilité}

L'objectif de l'étude de la sensibilité de l'échelle est de voir si elle est capable de mesurer des différences minimes entre individus ou chez le même individu au cours du temps (Bouletreau et al., 1999).

Tableau 3. Distribution des scores de l'ESDE

\begin{tabular}{|c|c|c|c|c|c|c|c|}
\hline Scores & 5 & 6 & 7 & 8 & 9 & 10 & 11 \\
\hline Effectifs & 3 & 11 & 8 & 10 & 33 & 42 & 48 \\
\hline Scores & 12 & 13 & 14 & 15 & 16 & 17 & 18 \\
\hline Effectifs & 48 & 43 & 32 & 37 & 42 & 44 & 49 \\
\hline Scores & 19 & 20 & 21 & 22 & 23 & 24 & 25 \\
\hline Effectifs & 48 & 68 & 22 & 18 & 19 & 4 & 15 \\
\hline
\end{tabular}

Tableau 4. Moyennes, écarts-type, symétrie et aplatissement des scores aux cinq items

\begin{tabular}{|c|c|c|c|c|}
\hline Items & $\mathbf{M}$ & $\mathbf{E . T}$ & Asy. & Apla. \\
\hline Je suis satisfait (e) de ma vie académique. & 3,36 & 1,35 & $-0,33$ & $-1,15$ \\
\hline $\begin{array}{c}\text { Mes conditions de vie scolaire sont excellentes. } \\
\text { Jusqu'à maintenant, j'ai obtenu les choses importantes } \\
\text { que je voulais dans le monde scolaire. }\end{array}$ & 3,04 & 1,32 & $-0,08$ & $-1,27$ \\
\hline $\begin{array}{c}\text { En général, ma vie académique correspond } \\
\text { de près à mes idéaux. }\end{array}$ & 2,98 & 1,32 & $-0,36$ & $-1,17$ \\
\hline $\begin{array}{c}\text { Si je pouvais recommencer mes études, je n'y } \\
\text { changerais presque rien. }\end{array}$ & 2,68 & 1,45 & $-0,10$ & $-1,34$ \\
\hline Satisfaction scolaire & 15,43 & 4,59 & $-0,03$ & $-1,36$ \\
\hline
\end{tabular}

D'après le Tableau 4, la distribution des scores présente un minimum $=5$, un maximum $=25$. Ensuite, les données descriptives des cinq items contenues dans le Tableau 4 montrent une moyenne de 15,43 et un écart-type de 4,59 des scores à l'échelle globale. De plus, les différentes moyennes indiquent qu'il n'y a pas d'effet de plafonnement ni d'effet de plancher. Les indices d'asymétrie et d'aplatissement sont presque proches de 0 et ne dépassent pas -2 et 2 . Nous pouvons conclure que la distribution des scores obtenus est normale comme l'on pourrait le constater sur la Figure 1 cidessous: 


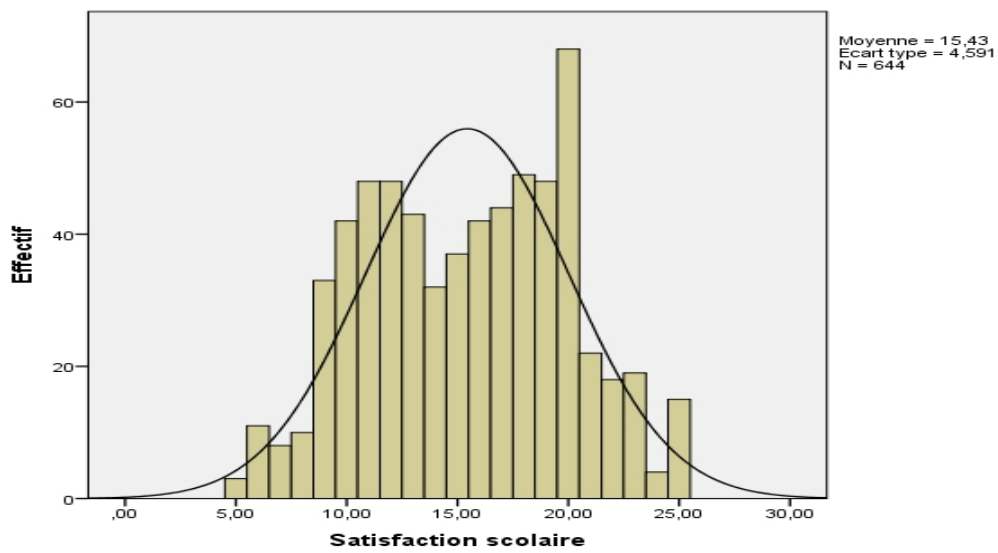

Figure 1. Histogramme et courbe de Gauss des scores de l'ESDE

\subsection{2. Étude de la fiabilité}

Pour vérifier cette homogénéité, 1'Alpha de Cronbach a été utilisé. Nous avons obtenu les scores suivants:

Tableau 5. Récapitulatif de traitement des observations de l'ESDE

\begin{tabular}{|c|c|c|}
\hline Observations & $\mathrm{N}$ & $\%$ \\
\hline Valide & 644 & 100,0 \\
\hline Exclus $^{\mathrm{a}}$ & 0 &, 0 \\
\hline Total & 644 & 100,0 \\
\hline
\end{tabular}

a. Suppression par liste basée sur toutes les variables de la procédure

Dans un premier temps, le récapitulatif de traitement des observations (Tableau 5) spécifie que $100 \%$ des 644 observations ont été retenues. Cela implique qu'il n'y avait aucune valeur manquante dans la base de données.

Tableau 6. Matrice de corrélations item-total

\begin{tabular}{|c|c|c|c|c|c|c|}
\hline & 1 & 2 & 3 & 4 & 5 & 6 \\
\hline $1 \mathrm{ESDE}$ & 1 & $0,63 * *$ & $0,79 * *$ & $0,78 * *$ & $0,70 * *$ & $0,56^{* *}$ \\
\hline 2 Item 1 & & 1 & $0,33 * *$ & $0,39 * *$ & $0,24 * *$ & $0,20 * *$ \\
\hline 3 Item 2 & & & 1 & $0,53 * *$ & $0,43 * *$ & $0,21 * *$ \\
\hline 4 Item 3 & & & & 1 & $0,51 * *$ & $0,22 * *$ \\
\hline 5 Item 4 & & & & & 1 & $0,21 * *$ \\
\hline 6 Item 5 & & & & & & 1 \\
\hline
\end{tabular}

La mise en relation de chaque item avec le score total de l'échelle montre de fortes corrélations, significatives au niveau 0,01 et comprises entre 0,56 et 0,79 (Tableau 6). La moyenne des coefficients de corrélation item-total est de 0,69. Cette valeur est autant forte que celle de $\alpha$ de Cronbach qui est de 0,70 pour les cinq éléments soumis (Tableau 7 ci-dessous). Toutes ces statistiques démontrent "un niveau d'homogénéité très acceptable dénotant peu de redondance entre les divers énoncés » (Vallerand \& Bissonnette, 1990, p. 299). 
Tableau 7. Cohérence interne de l'ESDE

\begin{tabular}{|c|c|}
\hline & Nombre d'éléments \\
\hline 0,704 & 5 \\
\hline
\end{tabular}

\subsection{3. Étude de la validité}

\subsubsection{Validité de contenu}

Cette étude vise à vérifier la validité de construit de notre épreuve de mesure, c'est-à-dire, voir "dans quelle mesure les divers aspects de cette épreuve sont représentatifs du concept visé » (Laveault et Grégoire, 1997 cité par Borteyrou et al., 2006, p. 3). Pour cela, l'analyse factorielle est la plus indiquée. Alors, avant de procéder à cette analyse, le coefficient de KaiserMeyer-Olkin $(\mathrm{KMO}=0,78)$ a été estimé et le test de sphéricité de Bartlett ( $\mathrm{p}$ $<.000$ ) appliqué. Ces statistiques indiquent bien que les corrélations entre les items de l'ESDE sont acceptables pour une analyse factorielle.

Tableau 8. Variance totale expliquée

\begin{tabular}{|c|c|c|c|c|c|c|}
\hline \multirow{2}{*}{$\begin{array}{c}\text { Compo- } \\
\text { santes }\end{array}$} & \multicolumn{3}{|c|}{ Valeurs propres initiales } & \multicolumn{3}{c|}{$\begin{array}{c}\text { Extraction Sommes des carrés } \\
\text { des facteurs retenus }\end{array}$} \\
\cline { 2 - 6 } & Total & $\begin{array}{c}\text { \% de la } \\
\text { variance }\end{array}$ & $\begin{array}{c}\% \\
\text { cumulés }\end{array}$ & Total & $\begin{array}{c}\text { \% de la } \\
\text { variance }\end{array}$ & $\begin{array}{c}\% \\
\text { cumulés }\end{array}$ \\
\hline 1 & 2,364 & 47,281 & 47,281 & 2,364 & 47,281 & 47,281 \\
\hline 2 & 0,878 & 17,551 & 64,832 & & & \\
\hline 3 & 0,774 & 15,488 & 80,320 & & & \\
\hline 4 & 0,555 & 11,106 & 91,427 & & & \\
\hline 5 & 0,429 & 8,573 & 100,000 & & & \\
\hline
\end{tabular}

À l'issue de l'analyse factorielle, il se dégage un seul facteur qui expliquerait à lui seul $47,28 \%$ de la variance, puisque c'est ce seul facteur celui qui a une racine caractéristique supérieur à un (Tableau 8). L'analyse en composantes principales avec rotation varimax que les 5 items de l'ESDE saturent sur un seul facteur (Tableau 9 ci-dessous) avec des valeurs de corrélation supérieures à 0,40 comme le recommande Gorsuch (1997). Ces résultats montrent à suffisance que les cinq items sont bien faits pour mesurer tous une même réalité qui n'est autre que la satisfaction dans les études.

Tableau 9. Matrices de composantes ${ }^{a}$

\begin{tabular}{|c|c|}
\hline \multirow{2}{*}{ Items } & Facteur \\
\cline { 2 - 2 } & 1 \\
\hline Je suis satisfait (e) de ma vie académique. & 0,822 \\
\hline Mes conditions de vie scolaire sont excellentes. & 0,765 \\
\hline $\begin{array}{c}\text { Jusqu'à maintenant, j'ai obtenu les choses importantes que je voulais dans } \\
\text { le monde scolaire. }\end{array}$ & 0,724 \\
\hline En général, ma vie académique correspond de près à mes idéaux. & 0,614 \\
\hline Si je pouvais recommencer mes études, je n'y changerais presque rien. & 0,449 \\
\hline
\end{tabular}

Méthode d'extraction : Analyse en composantes principales.

a. 1 composante extraite 


\subsubsection{Validité différentielle inter-sujets}

Tableau 10. Comparaison des scores moyens à l'ESDE selon le sexe et l'âge

\begin{tabular}{|c|c|c|c|c|}
\hline Caractéristiques & Effectif & Moyenne & $\begin{array}{l}\text { Écart } \\
\text {-type }\end{array}$ & ANOVA \\
\hline \multicolumn{4}{|c|}{ Sexe $(\mathrm{N}=642)$} & \multirow{3}{*}{$\begin{array}{c}\mathrm{t}=1,02 \\
\mathrm{p}=0,30\end{array}$} \\
\hline Féminin & 260 & 15,66 & 4,61 & \\
\hline Masculin & 382 & 15,29 & 4,59 & \\
\hline \multicolumn{4}{|c|}{$\operatorname{Age}(\mathrm{N}=644)$} & \\
\hline Adolescents & 300 & 15,87 & 4,47 & \multirow{2}{*}{$\begin{array}{l}t=2,25 ; \\
p=0,025\end{array}$} \\
\hline Adultes & 344 & 15,06 & 4,67 & \\
\hline
\end{tabular}

Même si l'écart d'effectifs entre les filles et les garçons semble considérable, ceux de la moyenne et de l'écart-type sont relativement faibles. D'où l'analyse de variance "sexe $\mathrm{X}$ échelle » ne révèle aucune différence significative. Par contre, avec un score moyen de 15,87 pour les élèves adolescents et 15,06 pour les élèves adultes, on obtient une différence significative, car pour $\mathrm{t}=2,25$ calculé, $\mathrm{p}$ observée $(0,025)$ est inférieure à $\mathrm{p}$ théorique $(0,05)$. Il y a donc une relation entre l'âge des élèves et la perception qu'ils ont du travail scolaire.

2.2.Rélation entre la satisfaction dans les études et le travail scolaire perçu

Tableau 11. ANOVA : Satisfaction dans les études et travail scolaire perçu

\begin{tabular}{|r|c|c|c|}
\hline $\begin{array}{c}\text { Dans l'ensemble, comment trouves- } \\
\text { tu ton travail scolaire ? }\end{array}$ & Effectifs & Moyenne & Écart-type \\
\hline Bon & 517 & 15,86 & 4,54 \\
\hline Mauvais & 127 & 13,71 & 4,41 \\
\hline Total & 644 & 15,43 & 4,59 \\
\hline $\mathrm{t}=4,81 \quad \mathrm{ddl}=642 \quad \mathrm{p}=0,000$ \\
\hline
\end{tabular}

Les élèves qui perçoivent leur travail à l'école comme « bon » sont plus nombreux que ceux qui le perçoivent comme «mauvais » (517 contre 127). Dans le même sens, ces mêmes élèves sont ceux qui présentent un score moyen à l'ESDE supérieur aux autres $(15,86$ contre 13,71$)$. Lorsqu'on a soumis ces différences aux analyses de variances, il ressort un $\mathrm{t}$ de Student significatif ( $\mathrm{t}$ $=4,81 ; p=0,000)$. Il est clair qu'il existe un lien entre la satisfaction dans les études et la perception du travail scolaire des sujets à l'étude.

\section{Discussion}

Cette étude avait un double objectif. D'une part, valider l'Échelle de Satisfaction dans les Études (ESDE) auprès des lycéens togolais et d'autre part, vérifier la relation entre la satisfaction scolaire et le travail scolaire perçu des mêmes élèves. Dans cette optique, et dans un premier temps, nos résultats au sujet de cette validation confirment l'hypothèse selon laquelle cette échelle présente de bonnes qualités psychométriques. La capacité de l'échelle à discriminer les sujets a été montrée par des statistiques satisfaisantes. La 
cohérence des items qui la composent a été soutenue par des corrélations itemstotal très fortes et un $\alpha$ de Cronbach acceptable. Enfin, la validité de contenu est démontrée par des analyses factorielles en composantes principales avec rotation varimax. Celles-ci ont confirmé le caractère unidimensionnel de l'échelle. Tous ces résultats corroborent parfaitement ceux obtenus par Vallerand et Bissonnette (1990) dans le contexte canadien lors de sa construction. Il va sans dire que cette échelle transcende les cultures et pourrait avoir une application en matière de prise de décision en orientation scolaire et de diagnostic de l'état de bien-être des élèves togolais.

S'agissant de la validité différentielle, les résultats aboutissent à l'idée qu'entre le sexe et l'âge, c'est l'âge qui constitue un facteur associé à la satisfaction scolaire; c'est-à-dire, que les plus âgés sont ceux qui ont un degré de satisfaction faible. Robin et Roux (2013) font le même constat, à savoir que plus l'âge augmente, plus le risque de ne pas aimer l'école augmente. Les élèves plus âgés ont toujours un risque plus important d'une satisfaction scolaire moindre par rapport aux plus jeunes, ceci à cause de leur parcours scolaire jonché d'échecs. Le sexe de l'élève n'est pas un facteur associé à la satisfaction scolaire. Ce résultat est contraire à celui d'Aldemir et Gülcan (2004) qui s'aperçoivent qu'en Turquie, tout porte à croire que l'appartenance au sexe féminin accroît la probabilité de satisfaction parce que la grande majorité des étudiantes $(88.1 \%)$ se déclaraient satisfaites de leur faculté contre $70 \%$ des étudiants. Contrairement à l'étude turque qui porte sur l'université, la nôtre a porté sur le secondaire où les enfants sont en pleine maturité liée au genre. La lutte contre les discriminations liées au genre engagées par l'État togolais dans les établissements scolaires rendrait la satisfaction presque indifférenciée chez les garçons et les filles.

Dans un second temps, l'étude confirme que plus les élèves sont satisfaits dans leurs études, plus ils perçoivent leur travail scolaire comme bon. Notre deuxième objectif est également atteint. De ce fait, nos résultats rejoignent ceux de Jorgensen, Fichten, et Havel (2012) pour lesquels la satisfaction globale était plus étroitement liée aux résultats scolaires. Même à l'université la satisfaction/l'insatisfaction manifestée à l'égard de l'université/ faculté a un impact sur les résultats de l'étudiant (Pike, 1991; Bean \& Bradley, 1986). Ce qui est important à souligner ici, c'est le sentiment de sécurité de l'élève dans son environnement. Si l'environnement de travail des élèves se construit sur la menace, les sentiments de peur et de méfiance cela risque de les entraîner vers l'agressivité et le rejet total de l'école, donc de l'insatisfaction scolaire. Un élève satisfait est très motivé à apprendre ses leçons, à accepter de changer ses perspectives, ses visions et donc de basculer dans la nouveauté et l'inconnu, de revoir ses mécanismes de réflexion. Il accepte facilement de savoir prendre des risques et d'accepter de tâtonner dans sa quête de réussite (Favre \& Favre, 1993). Ainsi, pour favoriser le développement de l'apprenant 
vers la réussite, il est donc nécessaire de mettre en place un environnement rassurant, juste et bienveillant pour ce dernier. C'est cet environnement ce qui influencera le système motivationnel de l'élève, lequel constitue l'instance régissant la qualité de son engagement et en bout de piste de son rendement scolaire (Pintrich, 2000; Zimmerman, 2000).

\section{Conclusion}

Dans un premier temps, les résultats auxquels nous sommes parvenus suggèrent que l'Échelle de Satisfaction Dans les Études élaborée dans le contexte canadien est adaptée au contexte togolais. Elle possède une sensibilité, une fidélité et une validité de contenu et différentielle acceptables. Elle est bien une échelle unidimensionnelle et destinée à mesurer la satisfaction scolaire. Dans un second temps, elle démontre l'importance de considérer la satisfaction scolaire dans la compréhension du travail scolaire des lycéens. Dans ce sens, cette étude apporte un nouveau facteur psychogénétique (la satisfaction scolaire) à la littérature scientifique togolaise basée sur le travail scolaire. Dans une perspective d'africanisation de cet outil, il serait intéressant d'envisager sa validation dans différents pays africains, en particulier au Burkina Faso. Par la même occasion, les études ultérieures pourraient s'intéresser aux facteurs sociaux (relations entre élèves) et pédagogiques (relation enseignant-étudiant; méthode pédagogique) pouvant influer positivement ou négativement sur la satisfaction scolaire.

\section{REMERCIEMENTS}

Au Professeur Robert J. Vallerand du Laboratoire de Recherche sur le Comportement Social (LRCS) de l'Université du Québec à Montréal (UQAM) pour avoir autorisé la validation de l'ESDE au Togo.

\section{References:}

1. Aldemir, C. \& Gülcan, Y. (2004). Satisfaction des étudiants dans l'enseignement supérieur en Turquie, Politiques et gestion de l'enseignement supérieur, 16 (2), 121-135.

2. Bacro, F., Ferrière, S., Florin, A., \& Guimard, P. (2014). Le bien-être des élèves à l'école et au collège: validation d'une échelle d'évaluation multidimensionnelle et analyses différentielles, rapport $\mathrm{n}^{\circ}$ 2013-013, appel à projet « L'égalité des chances à l'École », MENESR-DEPP.

3. Bean, J.P. \& Bradley, R.K. (1986). Untangling the SatisfactionPerformance relation for College Students. Journal of Higher Education, 57(4), 393-442.

4. Borteyrou, X., Rascle, N., Bruchon-Schweitzer, M. \& Collomb, P. (2006). Construction et validation d'une épreuve de groupe élaborée 
dans le cadre d'un « assessment center » pour des officiers de marine. L'orientation scolaire et professionnelle [En ligne], 35/4 |2006, mis en ligne le 15 décembre 2009, consulté le 10 septembre 2018. URL : http://osp.revues.org/1190 ; DOI : 10.4000/ osp.1190.

5. Bouletreau, A, Chouanière, D., Wild, P. \& Fontana, J.-M. (1999). Concevoir, traduire et valider un questionnaire A propos d'un exemple, EUROQUEST. Institut National de Recherche et de Sécurité. Disponible sur [URL] : http://lara.inist.fr/bitstream/handle/2332/1730/INRS_178.pdf?sequenc $\mathrm{e}=1$. (Consulté le 4 septembre 2018 à 13h55).

6. Favre, D. \& Favre, C. (1993). Un modèle complexe des motivations humaines : application à l'éclairage de la crise d'adolescence. Revue de Psychologie de la Motivation, 16, 27-42.

7. Gaudonville, T. (2017). L'impact de la qualité de vie sur les performances scolaires et le développement. Disponible sur [URL] : http://www.cnesco.fr/wpcontent/uploads/2017/10/170929_performan ces.pdf. (Consulté le 12 septembre 2018 à 15h03).

8. Gorsuch, R. L. (1997). Exploratory factor analysis: Its role in item analysis. Journal of Personality Assessment, 68(3), 532-560.

9. Hamner, W.C. \& Organ, W.D. (1978). Organizational Behaviour. Texas: Business Publications.

10. Huebner, E. (1991). Correlates of life satisfaction in children. School Psychology Quarterly, 6, 103-111.

11. Huebner, E. \& Alderman, G. (1993). Convergent and discriminant validation of a children's life satisfaction scale : Its relationship to selfand teacher-reported psychological problems and school functioning. Social indicators research 1(30), 71-82.

12. Jorgensen, S., Fichten, C. \& Havel, A. (2012). Satisfaction et réussite académique au cégep. Magazine électronique du CAPRES. Disponible sur [URL] : file:///F:/Satisfaction\%20Scolaire/2012-06-11-artS.Jorgensen.pdf. (Consulté le 10 septembre 2018 à 16h34).

13. Laveault, D. \& Grégoire, J. (1997). Introduction aux théories des tests en sciences humaines. Paris : De Boeck Université.

14. McCullough, G. \& Huebner, E. (2003). Life satisfaction reports of adolescents with learning disabilities and normally achieving adolescents. Journal of Psychoeducational Assessment 4(21), 311324.

15. Pike, G. R. (1991). The effects of background, coursework and involvement on students' grades and satisfaction. Research in Higher Education, 32,15-30.

16. Pintrich, P. R. (2000). The role of goal orientation in self-regulated learning. Dans M. Boekaerts, P. R. Pintrich \& M. Zeidner (Eds), 
Handbook of Self-Regulation. London / San Diego, CA : Academic Press.

17. Randolph, J., Kangas, M. \& Ruokamo, H. (2009). The preliminary development of the children's overall satisfaction with schooling scale (cosss). Child Indicator Resarch 1(2), 79-93.

18. Robin, S. \& Roux, M.-Th. (2013). Facteurs associés à la satisfaction scolaire parmi des groupes d'élèves en situation a priori défavorable. Etude réalisée à partir des données de l'enquête HBSC Poitiers 2007. Disponible sur [URL]: http://www.ors-poitoucharentes.org/pdf/NV66LBFondFrance13.pdf. (Consulté le 10 septembre 2018 à 17 h50).

19. Schreiner, L. A. \& Juillerat, S. L. (1994). Student satisfaction inventory. Iowa City, IA: Noel-Levitz Centers.

20. Vallerand, R. J. \& Bissonnette, R. (1990). Construction et validation de l'Echelle de Satisfaction dans les Etudes (ESDE). Canadian Journal of Behavioural Science, 22 (3), 295-306.

21. Zimmerman, B. J. (2000). Attaining self-regulation: a social cognitive perspective. In M. Boekaerts, P.R. Pintrich \& M. Zeidner (Eds), Handbook of Self-Regulation. London / San Diego, CA. 